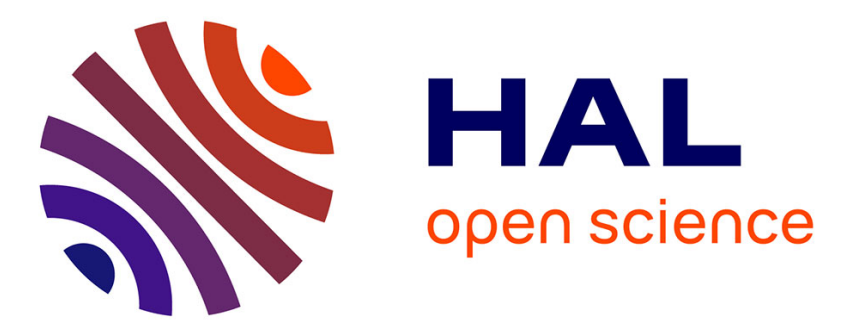

\title{
Modelling and Analysing Cloud Application Management
}

Antonio Brogi, Andrea Canciani, Jacopo Soldani

\section{To cite this version:}

Antonio Brogi, Andrea Canciani, Jacopo Soldani. Modelling and Analysing Cloud Application Management. 4th European Conference on Service-Oriented and Cloud Computing (ESOCC), Sep 2015, Taormina, Italy. pp.19-33, 10.1007/978-3-319-24072-5_2 . hal-01757559

\section{HAL Id: hal-01757559 \\ https://hal.inria.fr/hal-01757559}

Submitted on 3 Apr 2018

HAL is a multi-disciplinary open access archive for the deposit and dissemination of scientific research documents, whether they are published or not. The documents may come from teaching and research institutions in France or abroad, or from public or private research centers.
L'archive ouverte pluridisciplinaire HAL, est destinée au dépôt et à la diffusion de documents scientifiques de niveau recherche, publiés ou non, émanant des établissements d'enseignement et de recherche français ou étrangers, des laboratoires publics ou privés.

\section{(c)(1)}

Distributed under a Creative Commons Attribution| 4.0 International License 


\title{
Modelling and analysing cloud application management ${ }^{\star}$
}

\author{
Antonio Brogi, Andrea Canciani, and Jacopo Soldani \\ Department of Computer Science, University of Pisa, Italy
}

\begin{abstract}
Managing complex applications over heterogeneous clouds is one of the emerging problems in the cloud era. The OASIS Topology and Orchestration Specification for Cloud Applications (TOSCA) aims at solving this problem by providing a language to describe and manage complex cloud applications in a portable and vendor-agnostic way. TOSCA permits to define an application as an orchestration of components, whose types can specify states, requirements, capabilities and management operations - but not how they interact with each other.

In this paper we propose a simple extension of TOSCA that permits to specify the behaviour of management operations and their relations with states, requirements, and capabilities. We show how such an extension permits to automate various useful analyses, like determining the validity of a management plan, which are its effects, or which plans reach certain system configurations. Finally, we illustrate a proof-of-concept graphical interface that permits to edit and analyse management protocols in TOSCA applications.
\end{abstract}

\section{Introduction}

Cloud computing has revolutionized IT, by allowing to run on-demand distributed software systems at a fraction of the cost of just a few years ago. However, due to the lack of standardization, how to flexibly manage applications over heterogeneous clouds is still an open issue.

In this scenario, OASIS released TOSCA (Topology and Orchestration Specification for Cloud Applications [15[17]), a standard to support the automated management of complex cloud-based applications. TOSCA provides a modelling language to describe, in a portable and vendor-agnostic way, a cloud application and its management. An application is defined by instantiating component types, and by connecting a component's requirements to the capabilities of other components. Its management can then be described by orchestrating the operations of its components (like configure, install, start, etc.) into workflow plans.

Unfortunately, the current version of TOSCA [15] does not permit to specify the behaviour of a cloud application's management operations. More precisely, it is not possible to describe the order in which the management operations of a component must be invoked, nor how those operations depend on the requirements or how they affect the capabilities of that component (and hence

* Work partly supported by the European project SeaClouds (EU-FP7-ICT-610531). 
the requirements of other components they are connected to). This implies that the verification of whether a management plan is valid can only be performed manually, with a time-consuming and error-prone process.

In this paper we first propose a simple extension of TOSCA that permits to specify the behaviour of management operations and their relations with states, requirements, and capabilities. We define how to describe the management protocols of TOSCA components by means of finite state machines whose states and transitions are associated with conditions on the component's requirements and capabilities. Intuitively speaking, the objective of those conditions is to define the consistency of component's states and to constrain the executability of component's operations to the satisfaction of their requirements.

We then show how to the proposed extension of TOSCA permits to automate various analyses of management protocols, like determining whether management plans are valid, which are their effects, or which plans permit to reach certain system configurations.

Finally, we illustrate the feasibility of our approach by describing a proof-ofconcept web-based application that permits to edit the management protocols of TOSCA application components, and to analyse plans describing the management of a whole application.

The rest of the paper is organized as follows. Sect. 2 introduces TOSCA, while Sect. 3 illustrates a scenario motivating the need for an explicit, machinereadable representation of management protocols. Sect. 4 describes how TOSCA can be extended to model the behaviour of management operations, and how the proposed modelling permits to automate different types of analysis. Sect. 5 illustrates our proof-of-concept. Related work is discussed in Sect. 6, while some concluding remarks are drawn in Sect. 7 .

\section{Background: TOSCA}

TOSCA 15 is an emerging standard aimed at enabling the specification of portable cloud applications and the automation of their management. To do so, TOSCA provides a modelling language to describe the structure of a cloud application as a typed topology graph, and its tasks as plans. More precisely, each cloud application is represented as a ServiceTemplate (Fig. 1), consisting of a mandatory TopologyTemplate and of optional management Plans. Generic type definitions are also contained in the document defining the ServiceTemplate as they are referred to by the elements in its topology.

The TopologyTemplate is a typed directed graph describing the structure of the composite cloud application. Its nodes (NodeTemplates) model the application components, while its edges (RelationshipTemplates) model the relations among those components. NodeTemplates and RelationshipTemplates are typed by means of NodeTypes and RelationshipTypes, respectively. A NodeType defines (i) the observable properties of an application component, (ii) the possible states of its instances, (iii) its requirements, (iv) the capabilities it offers to satisfy other components' requirements, and (v) its management op- 


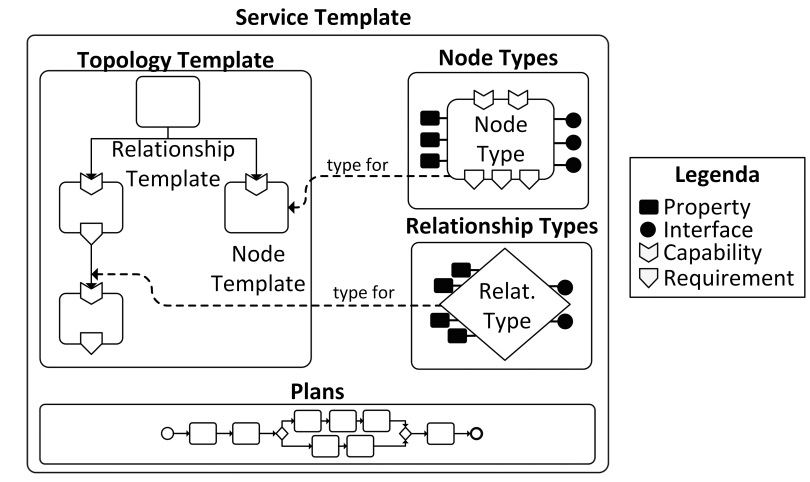

Fig. 1. TOSCA ServiceTemplate.

erations. RelationshipTypes describe the properties of relationships occurring among components. Syntactically, properties are described by PropertiesDefinition, states by InstanceStates, requirements by RequirementDefinitions (of certain RequirementTypes), capabilities by CapabilityDefinitions (of certain CapabilityTypes), and operations by Interfaces and Operations.

Plans instead allow to describe the management aspects of a ServiceTemplate. More precisely, each Plan is a workflow orchestrating the management Operations offered by the application components to address (part of) the management of the whole cloud application ${ }^{1}$.

\section{Motivating scenario}

Consider two utility web services, Translator and Convertor, and suppose that we want to manage them on a TOSCA-compliant cloud platform. After describing the services in TOSCA, we have to specify the third-party application components needed to properly host them. For instance, we may indicate that they have to run on an Apache server installed on a Debian operating system, which in turn runs on an VMWare virtual machine. Fig. 2 illustrates the resulting TopologyTemplate, according to the graphical notation introduced by Winery [14. For the sake of readability, we focus only on the lifecycle interfaces [8] of each NodeType instantiated in the topology (i.e., the interfaces containing the operations to install, configure, start, stop, and uninstall a component).

Suppose now that we want to specify the deployment of the Translator and Convertor services by writing a TOSCA Plan. It is worth noting that, since TOSCA does not include any representation of the management protocols of (third-party) NodeTypes, one may produce invalid Plans. For instance, while Fig. 3 illustrates three seemingly valid BPMN Plans, only (c) is a valid Plan. Plan (a) is not valid since Apache's Configure operation cannot be executed

\footnotetext{
${ }^{1}$ A more detailed and self-contained introduction to TOSCA can be found in [8].
} 


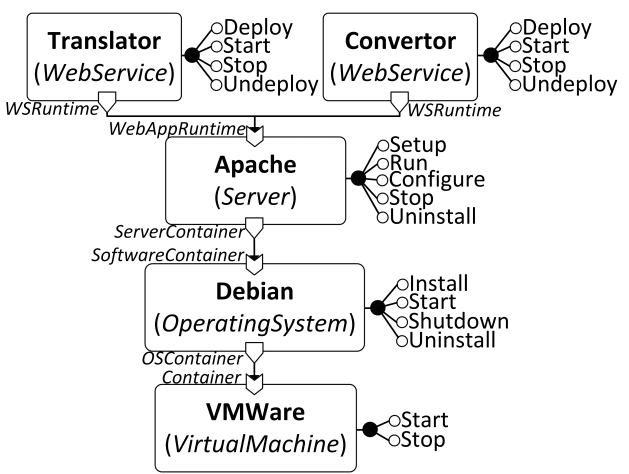

Fig. 2. Motivating scenario.

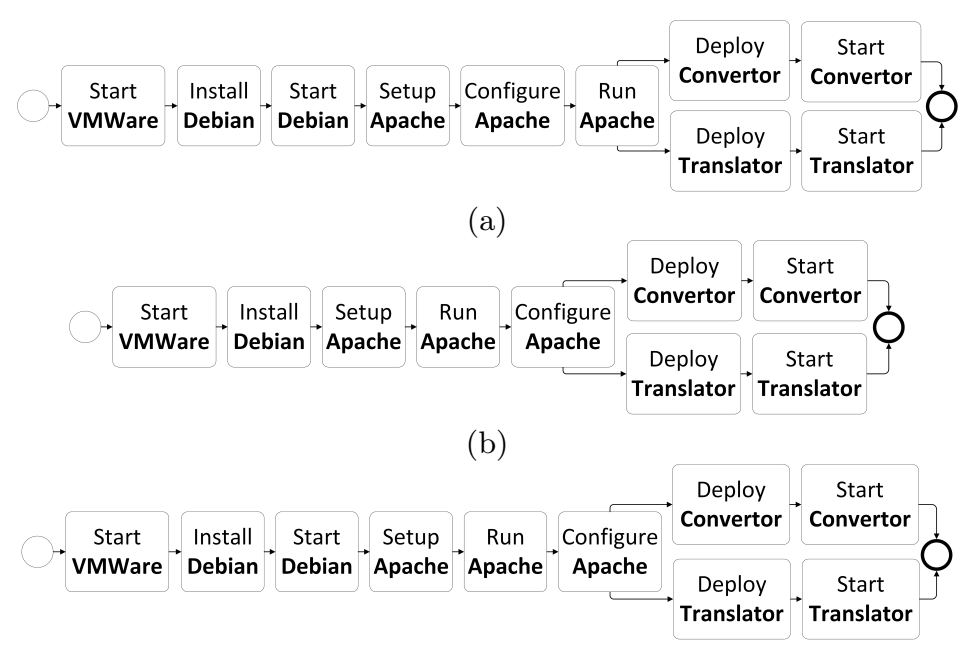

(c)

Fig. 3. Examples of deployment Plans.

before Apache itself is running, while Plan (b) is not valid since Apache cannot be installed if the Debian operating system is not running.

While the validity of Plans can be manually verified, this is a time-consuming and error-prone process. In order to enable the automated verification of the validity of Plans, TOSCA needs to be extended with an explicit, machine-readable representation of NodeTypes' management protocols.

\section{Management protocols for cloud applications}

TOSCA NodeTypes can be described by means of their states, requirements, capabilities, and management operations, but there is currently no way to specify 
how management operations affect states, how operations or states depend on requirements, or which capabilities are concretely provided in a certain state.

In this section we propose an extension of TOSCA that permits to specify the behaviour of management operations and their relations with states, requirements, and capabilities.

\subsection{Definition of management protocols}

Let $N$ be a TOSCA NodeType, and let us denote its states, requirements, capabilities, and management operations with $S_{N}, R_{N}, C_{N}$, and $O_{N}$, respectively.

We want to describe whether and how the management operations of $N$ depend on (i) other operations of the same node and/or on (ii) operations of other nodes providing the capabilities that satisfy the requirements of $N$.

(i) The first kind of dependencies can be easily described by specifying the relationship between states and management operations of $N$. More precisely, to describe the order with which the operations of $N$ can be executed, we introduce a transition relation $\tau$ specifying whether an operation $o$ can be executed in a state $s$, and which state is reached by executing $o$ in $s$.

(ii) The second kind of dependencies can be described by associating transitions and states with (possibly empty) sets of requirements to indicate that the corresponding capabilities are assumed to be provided. More precisely, the requirements associated with a transition $t$ specify which are the capabilities that must be offered to allow the execution of $t$. The requirements associated with a state of a NodeType $N$ specify which are the capabilities that must (continue to) be offered by other nodes in order for $N$ to (continue to) work properly.

To complete the description, we also associate to each state $s$ of a NodeType $N$ the capabilities provided by $N$ in $s$.

Definition 1. Let $N=\left\langle S_{N}, R_{N}, C_{N}, O_{N}, \mathcal{M}_{N}\right\rangle$ be a NodeType, where $S_{N}, R_{N}$, $C_{N}$, and $O_{N}$ are the finite sets of its states, requirements, capabilities, and management operations. $\mathcal{M}_{N}=\left\langle\bar{s}_{N}, \rho_{N}, \chi_{N}, \tau_{N}\right\rangle$ is the management protocol of $N$, where

$-\bar{s}_{N} \in S_{N}$ is the initial state,

- $\rho_{N}$ is a function indicating, for each state $s \in S_{N}$, which conditions on requirements must hold (i.e., $\rho_{N}(s) \subseteq R_{N}$ ),

- $\chi_{N}$ is a function indicating which capabilities of $N$ are concretely offered in a state $s \in S_{N}$ (i.e., $\chi_{N}(s) \subseteq C_{N}$ ), and

$-\tau_{N} \subseteq S_{N} \times 2^{R_{N}} \times O_{N} \times S_{N}$ is a set of quadruples modelling the transition relation (i.e., $\left\langle s, H, o, s^{\prime}\right\rangle \in \tau_{N}$ means that in state $s$, and if condition $H$ holds, o is executable and leads to state $\left.s^{\prime}\right)$.

Syntactically, to represent $\mathcal{M}_{N}$ we slightly extend the syntax ${ }^{2}$ for describing a TOSCA NodeType. First, we enrich the description of InstanceStates by

\footnotetext{
${ }^{2}$ A more detailed syntax for extended NodeTypes can be found in [5].
} 
introducing the nested elements ReliesOn and Offers. ReliesOn defines $\rho_{N}$ by enabling the association between states and conditions on requirements, while Offers defines $\chi_{N}$ by indicating the capabilities offered in a state. Furthermore, we introduce the element ManagementProtocol, to specify the InitialState $\bar{s}$ of a protocol, as well as the Transitions defining its transition relation $\tau_{N}$.

The management protocols of the NodeTypes in our motivating scenario (Sect. 3) are shown in Fig. 4 where $\mathcal{M}_{W S}$ is the management protocol for WebServices, $\mathcal{M}_{S}$ for Server, $\mathcal{M}_{O S}$ for OperatingSystem, and $\mathcal{M}_{V M}$ for VirtualMachine. Consider for instance the management protocol $\mathcal{M}_{S}$ of the Server

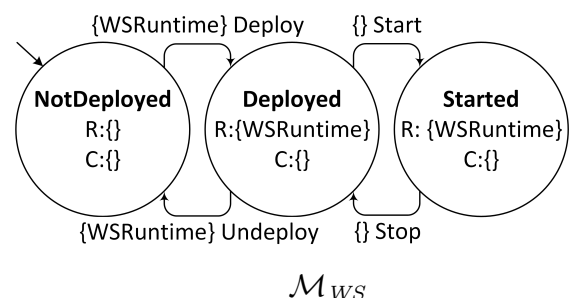

$\mathcal{M}_{W S}$

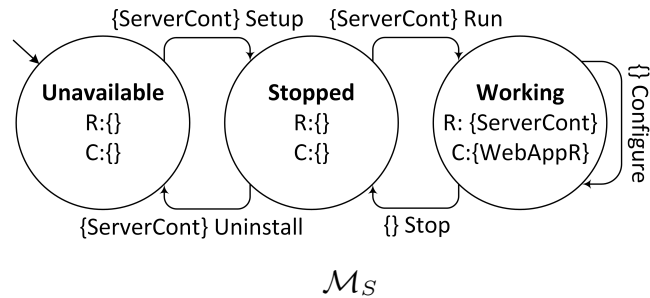

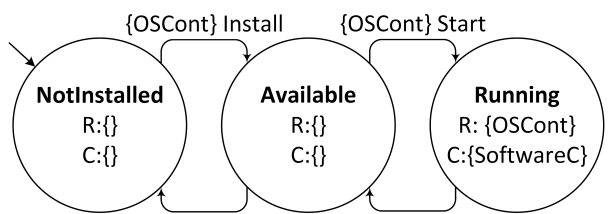

\{OSCont\} Uninstall \{OSCont\} Shutdown

$\mathcal{M}_{O S}$

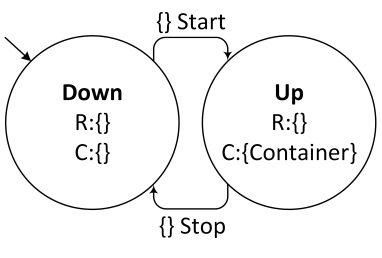

$\mathcal{M}_{V M}$

Fig. 4. Management protocols of the NodeTypes in our motivating scenario.

NodeType, typing a Tomcat server.Its states $S_{S}$ are Unavailable (initial), Stopped, and Working, the only requirement in $R_{S}$ is ServerContainer, the only capability in $C_{S}$ is WebAppRuntime, its management operations $O_{S}$ are Setup, Uninstall, Run, Stop, and Configure. States Unavailable and Stopped are not associated with any requirement or capability. State Working instead specifies that the capability corresponding to the ServerContainer requirement must be provided in order for Server to (continue to) work properly. State Working also specifies that Server provides the WebAppRuntime capability when in such state. Finally, all transitions (but those involving operations Stop and Configure) bind their executability to the availability of the capability that satisfies the ServerContainer requirement.

Management protocols (as per Def. 1) allow operations to have non-deterministic effects (e.g., a state may have two outgoing transitions corresponding to the same operation and leading to different states ${ }^{3}$ ). This form of non-determinism

\footnotetext{
${ }^{3}$ Note that the conditions of the two transitions may both hold even if the sets of requirements they refer to are disjoint. Hence the state obtained by performing the operation would be non-deterministic.
} 
is not acceptable when managing TOSCA applications 8]. We will thus focus on deterministic management protocols (i.e., protocols ensuring deterministic effects when performing an operation in a state).

Definition 2. Let $N=\left\langle S_{N}, R_{N}, C_{N}, O_{N}, \mathcal{M}_{N}\right\rangle$ be a NodeType. The management protocol $\mathcal{M}_{N}=\left\langle\bar{s}_{N}, \rho_{N}, \chi_{N}, \tau_{N}\right\rangle$ is deterministic if and only if

$$
\forall\left\langle s_{1}, H_{1}, o_{1}, s_{1}^{\prime}\right\rangle,\left\langle s_{2}, H_{2}, o_{2}, s_{2}^{\prime}\right\rangle \in \tau_{N}:\left(s_{1}=s_{2} \wedge o_{1}=o_{2}\right) \Rightarrow s_{1}^{\prime}=s_{2}^{\prime}
$$

\subsection{Analysis of management protocols}

In this section we describe different analyses that can be performed on the management protocol of a TOSCA application, such as checking the validity of a $\mathrm{Plan}$, determining its effects, or discovering Plans that allow to reach certain system configurations.

We first define an intensional operational semantics of the management protocol of a single component (viz., a TOSCA NodeType), which models all possible sequences of management operations that could be performed on a component if the conditions on the needed requirements were satisfied by the environment. Formally, the intensional semantics of the management protocol of a NodeType $N$ can be defined by a labelled transition system over configurations that are the states of $N$.

Definition 3. Let $N=\left\langle S_{N}, R_{N}, C_{N}, O_{N}, \mathcal{M}_{N}\right\rangle$ be a NodeType. The intensional semantics of the management protocol $\mathcal{M}_{N}$ of $N$ is modelled by a labelled transition system whose set of configurations is $S_{N}$ and where the transition relation is defined by the following inference rule:

$$
\begin{aligned}
& N=\left\langle S_{N}, R_{N}, C_{N}, O_{N}, \mathcal{M}_{N}\right\rangle \mathcal{M}_{N}=\left\langle\bar{s}_{N}, \rho_{N}, \chi_{N}, \tau_{N}\right\rangle \quad\left\langle s, H, o, s^{\prime}\right\rangle \in \tau_{N} \\
& s \stackrel{\langle H, o\rangle}{\longrightarrow}{ }_{N} s^{\prime}
\end{aligned}
$$

Intuitively, a transition $s \stackrel{\langle H, o\rangle}{\longrightarrow} N s^{\prime}$ denotes that operation $o$ can be executed on $N$ when $N$ is in state $s$, and under the hypothesis that condition $H$ holds, making $N$ evolve into state $s^{\prime}$.

The intensional semantics of the management protocol of a single NodeType permits to determine the conditions that must hold in the environment for sequences of management operations such as

$$
s_{0}{\stackrel{\left\langle H_{1}, o_{1}\right\rangle}{\longrightarrow}}_{N} s_{1}{\stackrel{\left\langle H_{2}, o_{2}\right\rangle}{\longrightarrow}}_{N} \cdots{\stackrel{\left\langle H_{h}, o_{h}\right\rangle}{\longrightarrow}}_{N} s_{h}
$$

to be effectively executable on a NodeTemplate of such NodeType.

We can now define the semantics of the management protocol of a whole application (viz., a TOSCA ServiceTemplate) by suitably composing the intensional semantics of the management protocols of the components (NodeTemplates) that form such application. Formally, the semantics of the management protocol 
of a ServiceTemplate $S$ can be defined by a labelled transition system over configurations that denote the states of the NodeTemplates of $S$. Intuitively, a transition

$$
G \stackrel{\left\langle o, N_{i}\right\rangle}{\longrightarrow}{ }_{S} G^{\prime}
$$

denotes that operation $o$ can be executed on NodeTemplate $N_{i}$ when the "global" state of $S$ is $G$, making $S$ evolve into the new global state $G^{\prime}$.

We first formally define the notion of global state of a ServiceTemplate and introduce a shorthand notation to denote the capability connected to a requirement in a ServiceTemplate (e.g., to denote Container as the capability connected to the OSContainer requirement in our motivating scenario - Fig. 22.

Definition 4. A global state of ServiceTemplate $S$ is denoted by a set

$$
\left\{\left(N_{1}, s_{1}\right), \ldots,\left(N_{m}, s_{m}\right)\right\}
$$

where $N_{1}, \ldots, N_{m}$ is the set of NodeTemplates in $S$, and where $s_{i}$ is a state of $N_{i}$. We denote by $\bar{G}$ the initial global state $S$ in which each NodeTemplate is in its initial state (viz., $\bar{G}=\left\{\left(N_{1}, \bar{s}_{1}\right), \ldots,\left(N_{m}, \bar{s}_{m}\right)\right\}$ ).

We also denote by $\operatorname{cap}_{S}(r)$ the (partial) function associating a requirement $r$ with the capability connected to $r$ in $S$ by means of a RelationshipTemplate.

We can now formally define the semantics of the management protocols in a ServiceTemplate $S$. Intuitively, a management operation $o$ can be executed on a NodeTemplate $N_{i}$ only if all the requirements needed by $N_{i}$ to perform $o$ are satisfied by the capabilities provided by (other) NodeTemplates in $S$.

Definition 5. The semantics of the management protocols in a ServiceTemplate $S$ is modelled by a labelled transition system whose configurations are the global states of $S$, and where the transition relation is defined by the following inference rule:

$$
\begin{gathered}
G=\left\{\left(N_{1}, s_{1}\right), \ldots,\left(N_{i}, s_{i}\right), \ldots,\left(N_{m}, s_{m}\right)\right\} \\
G^{\prime}=\left\{\left(N_{1}, s_{1}\right), \ldots,\left(N_{i}, s_{i}^{\prime}\right), \ldots,\left(N_{m}, s_{m}\right)\right\} \\
s_{i} \stackrel{\langle H, o\rangle}{\longrightarrow} N_{i} s_{i}^{\prime} \quad \forall r \in H: \operatorname{cap}_{S}(r) \text { is defined } \wedge \operatorname{cap}_{S}(r) \in \bigcup_{j=1}^{m} \chi_{N_{j}}\left(s_{j}\right) \\
G \stackrel{\left\langle o, N_{i}\right\rangle}{\longrightarrow}{ }_{S} G^{\prime}
\end{gathered}
$$

Definition 5 permits to model the evolution of a ServiceTemplate when a sequence of management operations is executed:

$$
G_{0} \stackrel{\left\langle o_{1}, N_{i_{1}}\right\rangle}{\longrightarrow} G_{1} G_{1} \stackrel{\left\langle o_{2}, N_{i_{2}}\right\rangle}{\longrightarrow} S \cdots \stackrel{\left\langle o_{h}, N_{i_{h}}\right\rangle}{\longrightarrow} G_{h} .
$$

It is worth observing that while Definition 5 checks that the requirements needed by a NodeTemplate $N_{i}$ to perform an operation $o$ are satisfied by the capabilities provided by the (other) NodeTemplates in $S$, it does not check whether after performing $o$ the requirements assumed by (the states of) all NodeTemplates will continue to be satisfied. We hence introduce the notion of consistent global state of a ServiceTemplate. 
Definition 6. A global state $\left\{\left(N_{1}, s_{1}\right), \ldots,\left(N_{m}, s_{m}\right)\right\}$ of a ServiceTemplate $S$ is consistent if and only if

$$
\forall i \in\{1 . . m\}, \forall r \in \rho_{N_{i}}\left(s_{i}\right): \operatorname{cap}_{S}(r) \text { is defined } \wedge \operatorname{cap}_{S}(r) \in \bigcup_{j=1}^{m} \chi_{N_{j}}\left(s_{j}\right) .
$$

Definitions 5 and 6 allow us to formally characterize the validity of a sequence of management operations.

Definition 7. A sequence $o_{1} o_{2} \ldots o_{n}$ of management operations is valid from a global state $G_{0}$ of a ServiceTemplate $S$ if and only if:

$$
G_{0} \stackrel{\left\langle o_{1}, N_{i_{1}}\right\rangle}{\longrightarrow} G_{1} \stackrel{\left\langle o_{2}, N_{i_{2}}\right\rangle}{\longrightarrow}{ }_{S} \cdots \stackrel{\left\langle o_{n}, N_{i_{n}}\right\rangle}{\longrightarrow}{ }_{S} G_{n}
$$

and each $G_{i}$ is a consistent global state.

The validity of a TOSCA Plan descends immediately from Def. 7 .

Definition 8. Let $G$ be a global state of a ServiceTemplate $S$. A Plan P for $S$ is valid from $G$ if and only if all its sequential traces are valid in $G$.

It is easy to see now that the deployment plan (c) of Fig. 3 is valid since, by starting from the initial global state, all its sequential traces are valid (and reach the same global state). Conversely, Plans (a) and (b) in Fig. 3 are not valid as their traces are not valid. More precisely, Plan (a) is not valid since all its

\begin{tabular}{|c|c|c|c|c|}
\hline VMWare & Debian & Apache & Translator & Convertor \\
\hline Down & NotInstalled & Unavailable & NotDeployed & NotDeployed \\
\hline \multicolumn{5}{|c|}{$\|$ VMare : Start } \\
\hline VMWare & Debian & Apache & Translator & Convertor \\
\hline$\underline{U p}$ & NotInstalled & Unavailable & NotDeployed & NotDeployed \\
\hline \multicolumn{5}{|c|}{$\|$ Debian : Install } \\
\hline VMWare & Debian & Apache & Translator & Convertor \\
\hline Up & Available & Unavailable & NotDeployed & NotDeployed \\
\hline \multicolumn{5}{|c|}{$\|$ Debian : Start } \\
\hline VMWare & Debian & Apache & Translator & Convertor \\
\hline Up & Running & Unavailable & NotDeployed & NotDeployed \\
\hline \multicolumn{5}{|c|}{$\|$ Apache : Setup } \\
\hline VMWare & Debian & Apache & Translator & Convertor \\
\hline Up & Running & Stopped & NotDeployed & NotDeployed \\
\hline
\end{tabular}
sequential traces produce the derivation shown in Fig. 5 , and Apache:Configure

Fig. 5. Initial evolution according to Plan (a) in Fig. 3

cannot be executed in the reached global state (because it requires Apache to be in state Working, instead of Stopped). On the other hand, Plan (b) is not valid since all its traces start as shown in Fig. 6, and Apache:Setup cannot be executed in the reached global state. It indeed requires the capability satisfying 


\begin{tabular}{|c|c|c|c|c|}
\hline VMWare & Debian & Apache & Translator & Convertor \\
\hline Down & NotInstalled & Unavailable & NotDeployed & NotDeployed \\
\hline \multicolumn{5}{|c|}{$\|$ VMare : Start } \\
\hline VMWare & Debian & Apache & Translator & Convertor \\
\hline$\underline{U p}$ & Notlnstalled & Unavailable & NotDeployed & NotDeployed \\
\hline \multicolumn{5}{|c|}{$\|$ Debian : Install } \\
\hline VMWare & Debian & Apache & Translator & Convertor \\
\hline Up & Available & Unavailable & NotDeployed & NotDeployed \\
\hline
\end{tabular}

Fig. 6. Initial evolution according to Plan (b) in Fig. 3

Apache's ServerContainer to be provided, but that capability is not provided when Debian is not in state Running.

The introduced modelling can be exploited for various other purposes besides checking Plans validity. For instance, valid Plans may not be enough, as their sequential traces may reach different global states. It is thus interesting to characterize deterministic Plans.

Definition 9. Let $G$ be a global state of a ServiceTemplate $S$. A valid Plan $P$ for $S$ is deterministic from $G$ if and only if all its sequential traces reach the same global state $G^{\prime}$.

It is also interesting to compute the effects of a valid Plan $P$ on the states of the components of a TOSCA ServiceTemplate, as well as on the requirements that are satisfied and the capabilities that are available. Such effects can be directly determined from the global state(s) reached by performing the sequential traces of $P$. Moreover, the problem of finding whether there is a deployment Plan which starts from the initial global state $\bar{G}$ and achieves a specific goal (e.g., bringing some components of an application to specific states or making some capabilities available) can be solved with a breadth-first search of the reachable global states. The same approach also works in the case of generic management plans (i.e., plans starting from a generic global state $G$ ), and it permits to find the sequential Plans (if any) allowing to reach a certain goal from whatever starting $G$. It also allows to characterize an interesting property that a ServiceTemplate may exhibit: if it is possible to reach the intial global state $\bar{G}$ from any $G$ that is reachable from $\bar{G}$ itself, then it is always possible to generate a plan for any (reachable) goal from any (reachable) global state. This ensures reversibility of actions, meaning that whatever $G$ we reach from $\bar{G}$, we can always get back to $\bar{G}$, thus always permitting a (soft) reset of the application.

\section{Proof-of-concept implementation}

We now illustrate the feasibility of our approach by introducing BARREL, a webbased application ${ }^{4}$ that permits to edit and analyse management protocols in

\footnotetext{
${ }^{4}$ BARREL's interface is written in HTML5, while its back-end is written in JavaScript. The application can be accessed at http://ranma42.github.io/MProt// with any modern web-browser, like Google Chrome or Mozilla Firefox. The source code is publicly available at https://github.com/ranma42/MProt
} 
TOSCA applications. In the following, we shall not deepen into implementation details, but rather focus on how BARREL can be used to edit and analyse existing TOSCA applications.

The very first step is to import a CSAR packag $\notin^{5}$ containing a ServiceTemplate, as well as the NodeTypes instantiated in its TopologyTemplate. Once the CSAR is loaded, the NodeTypes' names appear in the left hand pane of BARREL's interface (Available NodeTypes), and by selecting one of them the user can start editing its management protocol (Fig. 7). The management protocol

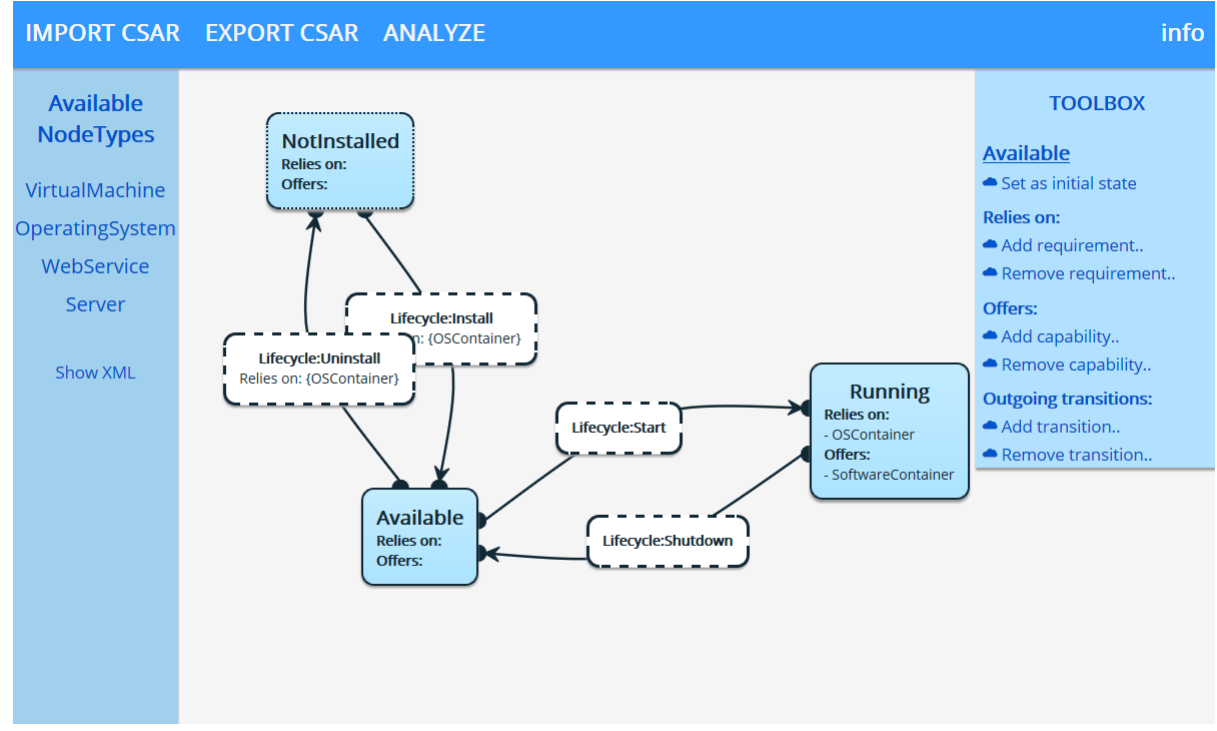

Fig. 7. Screenshot of BARREL: Editing mode.

is visualized in the central pane, by displaying the selected NodeType' states and the transitions among these states (if any). By clicking on a state $s$, a dedicated TOOLBOX opens in the right pane. This TOOLBOX permits editing the current values of $\rho(s), \chi(s)$, and $\tau(s)$, by allowing the user to update the set of requirements on which the selected state $s$ relies, the set of capabilities it offers, and its outgoing transitions. Such updates can also be viewed directly in the XML source of the current NodeType, by clicking on the Show XML button in the left pane. Once the NodeTypes' management protocols have been edited, the updated CSAR can be downloaded through the EXPORT CSAR functionality.

Users can also analyse the behaviour of the management operations appearing in the imported ServiceTemplate by selecting the ANALYZE option in the top menu. As a result, BARREL pops out a window showing the current global state of the application topology (Fig. 8). More precisely, the window lists all the

\footnotetext{
${ }^{5}$ A CSAR (Cloud Service ARchive) is a compressed zip file containing the TOSCA definitions describing the cloud application, along with the concrete artefacts implementing its components [15].
} 


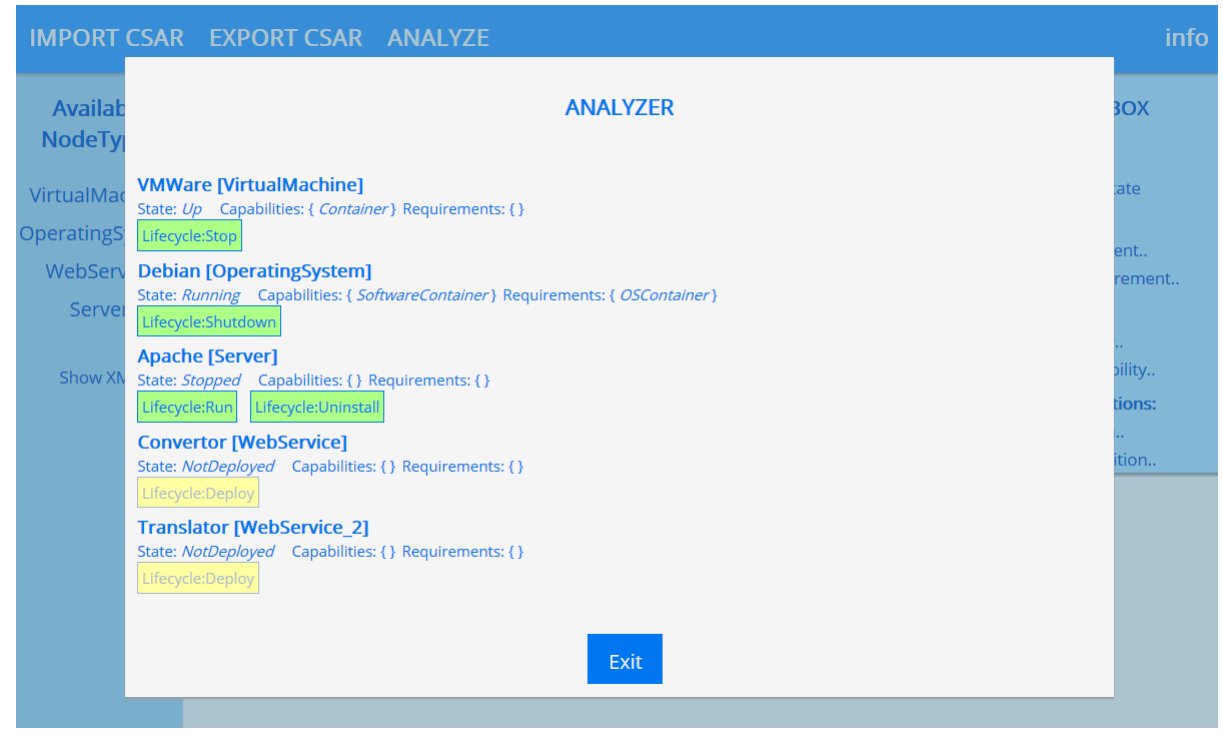

Fig. 8. Screenshot of BARrel: Analysis mode.

NodeTemplates in the TopologyTemplate, each associated with its current state, the requirements it relies on, the capabilities it offers and the operation actually available. Each operation is highlighted in green if all the capabilities connected to the requirements needed to execute it are currently available, otherwise it is highlighted in yellow. By clicking on a (green) operation users can simulate its execution, thus updating the current global state and then the ANALYZER window. If the reached state is inconsistent, a warning banner is displayed.

With the simple, interactive ANALYZER of BARREL, users can perform the analyses described in Sect. 4.2. For instance, to check whether a Plan is valid, they just need to simulate its sequential traces and check that no inconsistent state is traversed. They can also compute the effects of a valid Plan on states, capabilities and requirements by looking at the initial and final configurations displayed by the ANALYZER window. In this first version of BARREL, developers can only perform these analyses interactively, by manually clicking on the (green) operations and by looking at how they affect the global state ${ }^{6}$

It is worth noting that BARREL is already partially integrated with the OpenTOSCA open source ecosystem 3[14. BARREL is indeed able to process CSARs developed with the visual editor Winery [14, and it produces CSARs that can be imported in Winery ${ }^{7}$

${ }^{6}$ As part of our future work, we intend to extend BARREL in a working prototype capable of automatically performing all the aforementioned analyses.

7 While Winery imports the CSARs generated by Barrel, it does not properly process the information concerning management protocols. This is obviously because the extension to TOSCA we propose is not yet part of the TOSCA standard, and hence not (yet) supported in the OpenTOSCA open source environment. 


\section{Related work}

The problem of automating application management is well-known in computer science. In the cloud era, it has become even more prominent because of the complexity of both applications and platforms 9 . This is witnessed by the proliferation of so-called "configuration management systems", like Chef [10] or Puppet [18. These management systems provide domain-specific languages to model the desired configuration for a software solution, and employ a clientserver model to ensure that such configuration is met. However, the lack of a machine-readable representation of how to effectively manage cloud application components inhibits the possibility of performing automated analyses on components' configurations and dependencies.

A first attempt to model the deployment of cloud-based applications was the Aeolus component model [11. The Aeolus model shares our objective of describing various characteristics of cloud applications' components, including the possibility that component interfaces may vary depending on the internal component state. However, the Aeolus model only permits specifying what is offered and required in a state. Our approach instead allows developers to distinguish the requirements ensuring the consistency of a state from those constraining the applicability of a management operation. This permits to express transitions whose requirements concerns only the applicability of an operation and not the consistency of a state (e.g., the transition UUnavailable, \{ServerContainer\}, Setup, Stopped $\rangle$ of the protocol $\mathcal{M}_{S}$ in Fig. 44. Such kind of transitions cannot be directly modelled in Aelous (without introducing dummy intermediate states). Furthermore, Aelous and other emerging solutions, like Juju [13] or Engage [12, differ from our approach since so far they focus on the deployment of a cloud application, rather than on its whole management. Aelous, Juju, and Engage also differ from our approach since they are currently not integrated with any cloud interoperability standard.

TOSCA's rich type system has been exploited to devise various techniques that facilitate the the reuse of available services, like [47/19. Those techniques permit to match and adapt (fragments of) existing ServiceTemplates to implement a desired NodeType by checking that the features of the latter are all provided by the former. While those techniques are capable of overcoming various syntactical differences, they do not take into account the behaviour of management operations. Namely, they do not check whether the behaviour of a (fragment of) ServiceTemplate is compatible with the desired behaviour of a NodeType. As our proposal extends TOSCA's type system, it can be naturally exploited to extend the reuse techniques based on TOSCA, like [4]7]19], to account for management behaviour.

Finally, we have investigated the possibility of employing composition-oriented automata (like interface automata [1]) to model valid plans directly as the language accepted by the automaton obtained by composing the automata modelling the management protocols of the components of an application. The main drawbacks of such an approach are the size of the obtained automaton (which grows exponentially with the number of application components and 
hence makes the automaton scarcely readable even for simple applications), and the need of recomputing the automaton whenever a new component is added or its management protocol is modified.

\section{Conclusions}

In this paper we have proposed an extension of TOSCA to model the behaviour of management operations and their relations with states, requirements, and capabilities. We have then illustrated how such modelling permits to automate different analyses, such as determining whether a management $\mathrm{Plan}$ is valid, which are its effects, or which Plans allow to reach certain system configurations. To illustrate the feasibility of the proposed approach, we have developed a proof-of-concept graphical interface that permits to edit NodeTypes' management protocols and to analyze ServiceTemplates' Plans.

It is worth noting that, even if some of the behaviour-aware analyses discussed in Sect. 4.2 have exponential time complexity in the worst case, they still constitute a significant improvement with respect to the state-of-the-art, as currently the development and validation of Plans is performed manually, after delving through the documentation of the application's components.

It is also worth observing that our approach builds on top of, but is not limited to, TOSCA. It can indeed be adapted to other languages for specifying cloud applications (e.g., like CAMP [16] or GENTL [2]), and more in general to any stateful behaviour model of systems that describe states, requirements, capabilities, and operations.

We are currently investigating the possibility of modelling management protocols for cloud-based applications with Petri nets [6], with the objective of expressing some of the analyses described in Sect. 4.2 in terms of well-known Petri net notions (e.g., expressing Plan's validity in terms of firing sequences, or reducing Plan determination to coverability) and hence to possibly exploit some of the many available tools supporting the analyses of Petri nets. We see two other directions for immediate future work. On the one hand, we intend to extend our proof-of-concept BARREL into a working prototype supporting all the analyses described in Sect. 4.2 and to fully integrate it with the OpenTOSCA open source environment 3]14. On the other hand, as we anticipated in Sect. 6, another interesting direction for future work is to extend the matching

and adaptation reuse techniques based on TOSCA [4/7/19] to take into account the management behaviour of cloud-based applications.

\section{References}

1. de Alfaro, L., Henzinger, T.A.: Interface automata. In: Proceedings of the 8th European Software Engineering Conference Held Jointly with 9th ACM SIGSOFT International Symposium on Foundations of Software Engineering. pp. 109-120. ESEC/FSE-9, ACM (2001) 
2. Andrikopoulos, V., Reuter, A., Sáez, S.G., Leymann, F.: A GENTL Approach for Cloud Application Topologies. In: Villari, M., Zimmermann, W., Lau, K.K. (eds.) Service-Oriented and Cloud Computing. LNCS, vol. 8745, pp. 148-159. Springer (2014)

3. Binz, T., Breitenbücher, U., Haupt, F., Kopp, O., Leymann, F., Nowak, A., Wagner, S.: OpenTOSCA - a runtime for TOSCA-based cloud applications. In: Basu, S., Pautasso, C., Zhang, L., Fu, X. (eds.) Service-Oriented Computing. LNCS, vol. 8274, pp. 692-695. Springer (2013)

4. Brogi, A., Soldani, J.: Matching cloud services with TOSCA. In: Canal, C., Villari, M. (eds.) Advances in Service-Oriented and Cloud Computing, CCIS, vol. 393, pp. 218-232. Springer (2013)

5. Brogi, A., Canciani, A., Soldani, J.: Modelling the behaviour of management operations in TOSCA. Tech. Rep., University of Pisa (July 2015)

6. Brogi, A., Canciani, A., Soldani, J., Wang, P.: Modelling the behaviour of management operations in cloud-based applications. In: Moldt, D. (ed.) Proceedings of the International Workshop on Petri Nets and Software Engineering, PNSE'15. CEUR Workshop Proceedings, vol. 1372, pp. 191-205. CEUR-WS.org (2015)

7. Brogi, A., Soldani, J.: Reusing cloud-based services with TOSCA. In: INFORMATIK 2014, Lecture Notes in Informatics (LNI). vol. 232, pp. 235-246. Gesellschaft für Informatik (GI) (2014)

8. Brogi, A., Soldani, J., Wang, P.: TOSCA in a Nutshell: Promises and Perspectives. In: Villari, M., Zimmermann, W., Lau, K.K. (eds.) Service-Oriented and Cloud Computing. LNCS, vol. 8745, pp. 171-186. Springer (2014)

9. Buyya, R., Yeo, C.S., Venugopal, S., Broberg, J., Brandic, I.: Cloud computing and emerging IT platforms: Vision, hype, and reality for delivering computing as the 5th utility. Future Generation Computer Systems 25(6), 599 - 616 (2009)

10. Chef: Opscode. https://www.opscode.com/chef

11. Di Cosmo, R., Mauro, J., Zacchiroli, S., Zavattaro, G.: Aeolus: A component model for the cloud. Information and Computation 239(0), 100 - 121 (2014)

12. Fischer, J., Majumdar, R., Esmaeilsabzali, S.: Engage: A deployment management system. In: Proceedings of the 33rd ACM SIGPLAN Conference on Programming Language Design and Implementation. pp. 263-274. PLDI '12, ACM (2012)

13. Juju: DevOps distilled. https://juju.ubuntu.com

14. Kopp, O., Binz, T., Breitenbücher, U., Leymann, F.: Winery - Modeling Tool for TOSCA-based Cloud Applications. In: Proceedings of the 11th International Conference on Service-Oriented Computing. Springer (2013)

15. OASIS: Topology and Orchestration Specification for Cloud Applications. http: //docs.oasis-open.org/tosca/TOSCA/v1.0/TOSCA-v1.0.pdf (2013)

16. OASIS: Cloud Application Management for Platforms (CAMP). http://docs. oasis-open.org/camp/camp-spec/v1.1/camp-spec-v1.1.pdf (2014)

17. OASIS: TOSCA Simple Profile in YAML. http://docs.oasis-open.org/tosca/ TOSCA-Simple-Profile-YAML/v1.0/TOSCA-Simple-Profile-YAML-v1.0.pdf (2014)

18. Puppet: Puppet labs. https://puppetlabs.com

19. Soldani, J., Binz, T., Breitenbücher, U., Leymann, F., Brogi, A.: TOSCA-MART: A method for adapting and reusing cloud applications. Tech. Rep., University of Pisa (March 2015) 